\title{
Control of Untethered Magnetically Actuated Tools with Localization Uncertainty using a Rotating Permanent Magnet
}

\author{
Arthur W. Mahoney and Jake J. Abbott
}

\begin{abstract}
Magnetically actuated tools (MATs) that utilize rotating magnetic fields for propulsion, such as active capsule endoscopes and magnetic microrobots, have typically been controlled using either arrangements of electromagnets or permanent-magnet systems operated in limited configurations. It was recently shown that a rotating magnetic field for MAT actuation can be generated using a single rotating permanent magnet (RPM) from any position in space with a unique axis of rotation. The method has potential benefits for clinical systems, but it requires knowledge of the MAT position with respect to the RPM. In any application, MAT localization will be subject to uncertainty caused by sensor noise, slow update rates, and/or localization failure. In this paper, we develop and experimentally verify worst-case bounds on properties of the rotating dipole field, given a worst-case bound on localization error, which can be used to design operating procedures that mitigate undesired MAT behavior in the presence of known localization uncertainty. The results are important for the robust operation of rotating MATs actuated using a single rotating permanent magnet in a clinical setting.
\end{abstract}

\section{INTRODUCTION}

Untethered magnetic devices, such as magnetic microrobots [1] and active capsule endoscopes [2], have become an active area of research because of their potential impact to minimally invasive medicine. These devices typically consist of a rigidly attached magnetic body on which magnetic forces and torques are applied by an external field. Due to the difficulty of scaling electromagnetic systems to clinical sizes, researchers are considering the use of permanent magnets for actuation. Some of these approaches utilize magnetic forces for pulling [3], while others apply torque generated by rotating magnetic fields to roll on a surface [4], [5], or crawl through a lumen via helical propulsion [6]. Because these devices can be viewed as simple end-effectors of a larger robotic system, and they may range in size from the microscale to the mesoscale, we refer to them herein as magnetically actuated tools (MATs) without any implied size.

In prior work, MATs actuated with a rotating permanent magnet (RPM) have been constrained to operated in radial or axial positions relative to the RPM (Fig. 1) where the rotation of the field is easy to visualize [4]-[6]. In [7], we show that MATs can be successfully actuated by an RPM in any position, removing the need to be exclusively operated in axial or radial configurations. The methods presented in [7], however, require the MAT position in space to be

This material is based upon work supported by the National Science Foundation under Grant Nos. 0952718 and 0654414.

A. W. Mahoney is with the School of Computing, and J. J. Abbott is with the Department of Mechanical Engineering, University of Utah, Salt Lake City, UT, 84112 USA. art.mahoney@utah. edu

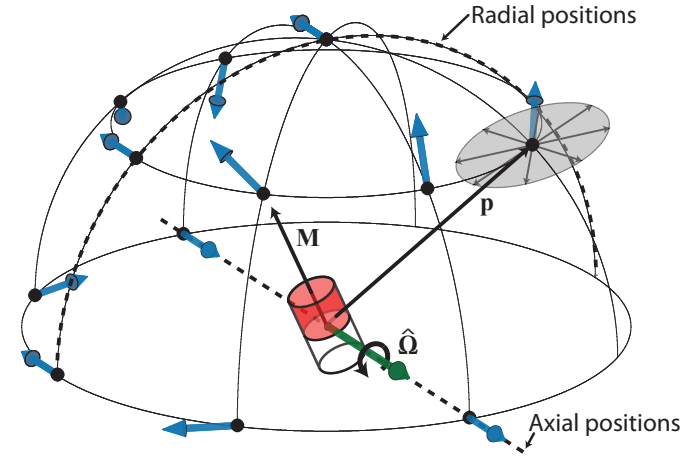

Fig. 1. When a dipole magnet with moment $\mathbf{M}$ rotates around the axis $\hat{\Omega}$ with $\mathbf{M}$ perpendicular to $\hat{\Omega}$, the field vector at any given position rotates around, and is perpendicular to, a constant axis $\hat{\omega}$. The axis $\hat{\omega}$ at various positions are illustrated with large blue arrows. A representation of the ellipse traced out by the rotating magnetic field at the position $\mathbf{p}$ is shown. Any position on the $\hat{\boldsymbol{\Omega}}$ axis is denoted to be in an axial position and any position in the plane spanned by the rotating $\mathbf{M}$ is a radial position.

known. A variety of localization strategies exist. The most appropriate depends on the MAT size and the environment in which it operates. If the MAT is visible, direct visualization approaches can be applied [1]. If not, RF triangulation with $\sim 37 \mathrm{~mm}$ error [8], magnetic position localization strategies that measure the magnetic field generated by the RPM (or MAT) with $\sim 10 \mathrm{~mm}$ error [9], [10], or computer vision approaches using images obtained through CT scan or x-ray fluoroscopy with $\sim 1 \mathrm{~mm}$ error [11] may be applied. Each method will be subject to uncertainty caused by noise, slow update rates, and/or temporary failure or obstruction.

When actuating a MAT with the nonuniform field produced by a single RPM, the applied field magnitude, rotation axis, and instantaneous rotational velocity all vary depending on the placement of the MAT relative to the RPM. If the rotation axis and velocity of the RPM are set according to [7] to produce a desired rotating magnetic field at an expected MAT position, then variation of the MAT position from expected will cause the rotating field experienced by the MAT to deviate from expected. Individual MATs may respond differently to unintended deviation in the rotating magnetic field depending on their design and the environment in which they operate. However, it is likely that unexpected deviation will produce undesired MAT behavior, and in the worst-case may result in loss of control authority.

In this paper, we present analysis of the worst-case deviation of applied field magnitude, rotation axis, and instantaneous rotational velocity from that expected, given a known worst-case bound on localization error, which can 
be used to design RPM operating procedures that reduce unexpected MAT behavior in the presence of known localization uncertainty. This paper only studies how the behavior of the rotating dipole field, generated by a single RPM, varies according to position, making the results applicable to any rotating MAT including active capsule endoscopes and magnetic microrobots. We demonstrate our results by measuring the magnetic field and by actuating a threaded MAT shaped like a wireless endoscope capsule with artificially induced uncertainty.

\section{Controlling Local Field Rotation}

The results of [7] readily enable the solution to be found for the necessary RPM rotation axis that produces an applied rotating field about any given rotation axis at any given position relative to the RPM. These results are necessary for Sec. III and are reviewed here in an abbreviated form.

All vectors are expressed in a common, static coordinate frame, and the "hat" symbol denotes a vector normalized to unit length. Let the RPM's dipole moment $\mathbf{M}$ rotate around the axis $\hat{\Omega}$ such that $\mathbf{M}$ is always perpendicular to $\hat{\Omega}$, and the direction of rotation is found using a right-hand rule. Let the vector $\hat{\omega}$ be the desired rotation axis of the local magnetic field at the MAT position p. Assuming the magnetic field $\mathbf{H}$, generated by the RPM, at position $\mathbf{p}$ can be accurately modeled with the point-dipole model, given by

$$
\mathbf{H}=\frac{1}{4 \pi|\mathbf{p}|^{3}}\left(3 \hat{\mathbf{p}} \hat{\mathbf{p}}^{\top}-\mathbb{I}\right) \mathbf{M}=\frac{1}{4 \pi|\mathbf{p}|^{3}} \mathbb{H} \mathbf{M},
$$

where $\mathbb{I}$ is the identity matrix and $\hat{\mathbf{p}}$ is the unit vector in the direction of $\mathbf{p}$, then the necessary $\hat{\Omega}$ that achieves $\hat{\boldsymbol{\omega}}$ at $\mathbf{p}$ is

$$
\hat{\Omega}=\widehat{\mathbb{H} \hat{\omega}} \text {. }
$$

The matrix $\mathbb{H}$ is always invertible and the solution for the local field axis of rotation $\hat{\omega}$ at the position $\mathbf{p}$, given the RPM axis of rotation $\hat{\Omega}$, is found with

$$
\hat{\omega}=\widehat{\mathbb{H}^{-1} \hat{\Omega}} \text {. }
$$

where $\mathbb{H}^{-1}=(\mathbb{H}-\mathbb{I}) / 2$, requiring no matrix inversion.

At any given MAT position, the instantaneous field magnitude $|\mathbf{H}|$ fluctuates in an elliptical fashion as the RPM rotates about $\hat{\Omega}$, and is given by

$$
|\mathbf{H}|=\frac{|\mathbf{M}|}{4 \pi|\mathbf{p}|^{3}} \sqrt{1+3\left(\hat{\mathbf{M}}^{\top} \hat{\mathbf{p}}\right)^{2}}
$$

and the minimum and maximum field magnitudes are

$$
\begin{aligned}
|\mathbf{H}|_{\min } & =\frac{|\mathbf{M}|}{4 \pi|\mathbf{p}|^{3}} \\
|\mathbf{H}|_{\max } & =\frac{|\mathbf{M}|}{4 \pi|\mathbf{p}|^{3}} \sqrt{1+3|\tilde{\mathbf{p}}|^{2}}
\end{aligned}
$$

where the vector $\tilde{\mathbf{p}}=\left(\mathbb{I}-\hat{\mathbf{\Omega}} \hat{\Omega}^{\top}\right) \hat{\mathbf{p}}$ is the projection of $\hat{\mathbf{p}}$ onto the plane perpendicular to $\hat{\Omega}$ (i.e., the plane in which $\mathbf{M}$ resides). The maximum field magnitude occurs when $\mathbf{M}$ is parallel to $\tilde{\mathbf{p}}$ and the minimum field magnitude occurs when $\mathbf{M}$ is perpendicular to $\tilde{\mathbf{p}}$. $|\mathbf{H}|_{\max }$ is, at most, twice $|\mathbf{H}|_{\min }$.
The instantaneous angular velocity of the applied field varies through each RPM cycle as well. If $\boldsymbol{\omega}$ and $\boldsymbol{\Omega}$ denote the instantaneous angular velocities of the local field and the RPM, respectively, then $|\boldsymbol{\omega}|$ and $|\boldsymbol{\Omega}|$ are related by

$$
|\boldsymbol{\omega}|=\left(\frac{|\mathbf{H}|_{\min }|\mathbf{H}|_{\max }}{|\mathbf{H}|^{2}}\right)|\boldsymbol{\Omega}| \text {. }
$$

The position $\mathbf{p}$ of the MAT relative to the RPM is measured by the localization system. With $\mathbf{p}$ known and the desired applied field rotation axis $\hat{\omega}$ for MAT actuation known, the necessary actuator axis of rotation $\hat{\Omega}$ can be quickly found by (2). As the MAT or the RPM moves and the direction of $\mathbf{p}$ changes, (2) must be updated by repacking the matrix $\mathbb{H}$, and a new solution for $\hat{\Omega}$ must be produced to maintain the desired field rotation axis at $\mathbf{p}$. This approach to MAT actuation is demonstrated in [7].

\section{Controlling Rotating MATs With LOCALIZATION UNCERTAINTY}

Any method used for MAT localization will produce an expected MAT position $\overline{\mathbf{p}}$ with an associated uncertainty. In practice, the expected position will be used in (2), (4), and (7) to generate expected (and desired) applied field rotation behavior for MAT actuation. In nonuniform fields generated by an RPM, the local field rotation axis, the instantaneous field magnitude, and the instantaneous field rotation speed vary depending on the MAT position p. Error between the expected MAT position $\overline{\mathbf{p}}$ and the actual MAT position p, when applying (2), (4), and (7) for control, may cause undesired MAT behavior. We present worst-case bounds on all three factors above if the MAT is known to reside in a ball of radius $r$, centered at the expected position $\overline{\mathbf{p}}$, assuming that $r<|\overline{\mathbf{p}}|$ (i.e., the RPM is not within the ball) and that the RPM field can be modeled with the point-dipole model.

We find that the three factors can be non-dimensionalized and purely described by the parameters $\bar{\theta}$ and $r /|\overline{\mathbf{p}}|$, where $\bar{\theta}$ measures the angle between the expected MAT position $\overline{\mathbf{p}}$ and the RPM rotation axis $\hat{\mathbf{\Omega}}$, and the ratio $r /|\overline{\mathbf{p}}|$ describes the relative uncertainty in the MAT position. Because (2), (4), and (7) are radially symmetric about the axis $\hat{\Omega}$, the three factors are invariant to the rotation of $\overline{\mathbf{p}}$ about $\hat{\Omega}$, so considering $r /|\overline{\mathbf{p}}| \in[0,1)$ and $\bar{\theta} \in\left[0^{\circ}, 180^{\circ}\right]$ captures all three parameters for any expected position $\overline{\mathbf{p}}$.

\section{A. Worst-case Rotation Axis Misalignment}

Let the set $\mathcal{B}$ hold every position in the ball centered at $\overline{\mathbf{p}}$, known to contain the MAT. If $\hat{\boldsymbol{\Omega}}$ remains constant, the rotation axis $\hat{\boldsymbol{\omega}}$ of the rotating applied field, given by (3), varies at positions throughout $\mathcal{B}$. Let $\hat{\bar{\omega}}$ be the expected rotation axis at the expected MAT position $\overline{\mathbf{p}}$, and let $\psi$ measure the angle between $\hat{\overline{\boldsymbol{\omega}}}$ and any other rotation axis in $\mathcal{B}$. The maximum $\psi$ in the ball is denoted by $\max _{\mathcal{B}}(\psi)$. In this notation, the subscript of max denotes the set over which the argument is maximized.

For any given RPM rotation axis $\hat{\Omega}$, the expected local field rotation axis $\hat{\overline{\boldsymbol{\omega}}}$ is given by (3) with the matrix $\mathbb{H}^{-1}$ packed with the expected position $\overline{\mathbf{p}}$. The rotation axis at 
(a)

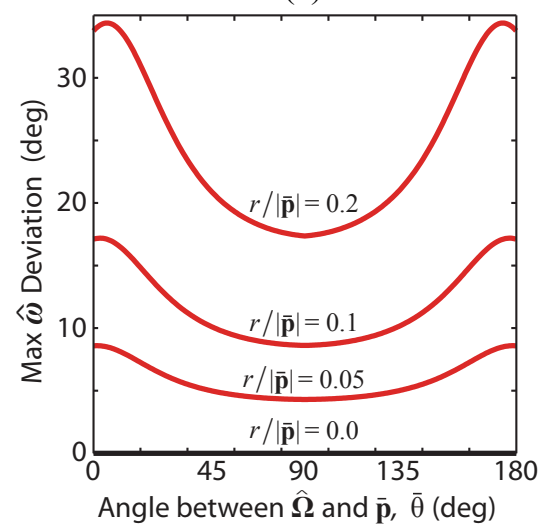

(b)

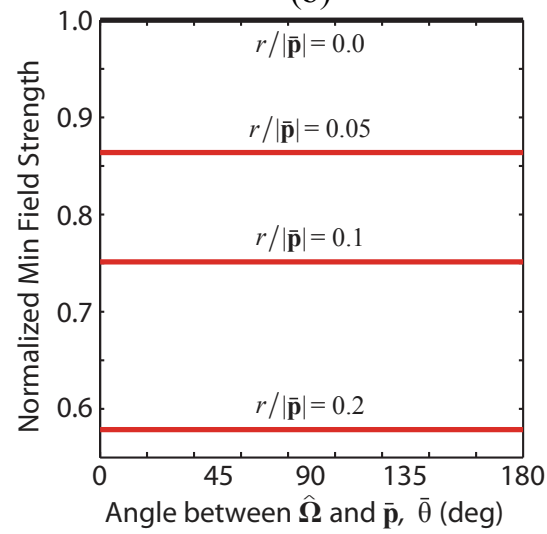

(c)

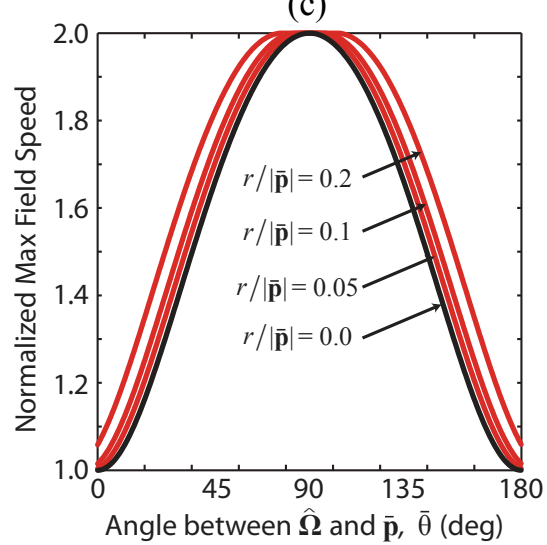

Fig. 2. If the MAT is known to reside in a ball of radius $r$ centered at an expected position $\overline{\mathbf{p}}$, the worst-case deviation of $\hat{\boldsymbol{\omega}}$ from expected $\hat{\overline{\boldsymbol{\omega}}}$ within the ball is shown in (a), the worst-case minimum field magnitude (normalized by $|\mathbf{M}| / 4 \pi|\overline{\mathbf{p}}|^{3}$ ) within the ball is shown in (b), and the worst-case maximum local field rotation speed (normalized by $|\boldsymbol{\Omega}|$ ) in the ball is shown in (c). In all three plots, the expected position $\overline{\mathbf{p}}$ is varied such that the angle between $\overline{\mathbf{p}}$ and $\hat{\boldsymbol{\Omega}}$, denoted by $\bar{\theta}$ ranges from $0^{\circ}$ to $180^{\circ}$. The effect of increasing the uncertainty relative to the expected distance between the RPM and MAT (i.e., increasing $r /|\overline{\mathbf{p}}|)$ is shown in each plot with $r /|\overline{\mathbf{p}}|=\{0.0,0.05,0.1,0.2\}(r /|\overline{\mathbf{p}}|=0.0$ corresponds to no uncertainty).

some other position $\mathbf{p}$ in $\mathcal{B}$ is given by (3). An analytic solution for $\max _{\mathcal{B}}(\psi)$ is nontrivial, however, $\max _{\mathcal{B}}(\psi)$ can be found numerically for any given $r$ and $\overline{\mathbf{p}}$ by comparing $\hat{\boldsymbol{\omega}}$ to $\hat{\overline{\boldsymbol{\omega}}}$ at every enumerated position in $\mathcal{B}$.

Fig. 2(a) shows four plots of $\max _{\mathcal{B}}(\psi)$ for $r /|\overline{\mathbf{p}}|=$ $\{0.0,0.05,0.1,0.2\}$. As $r /|\overline{\mathbf{p}}|$ increases, the worst-case deviation of $\hat{\boldsymbol{\omega}}$ from $\hat{\bar{\omega}}$ also tends to increase. It is also evident from the figure that the commanded $\hat{\omega}$ is more sensitive to localization uncertainty in axial positions than radial positions (see Fig. 1). If the proper alignment of $\hat{\omega}$ with $\hat{\bar{\omega}}$ is critical for MAT control, then the RPM should be positioned near radial positions or $|\overline{\mathbf{p}}|$ should be increased to make $r /|\overline{\mathbf{p}}|$ small. Increasing $|\overline{\mathbf{p}}|$ comes at the cost of decreasing the expected field magnitude, however.

\section{B. Worst-case Field Magnitude}

The instantaneous magnitude of the rotating magnetic field is given by (4). At a given MAT position $\mathbf{p}$ in $\mathcal{B}$, the field magnitude ranges from $|\mathbf{H}|_{\min }$ to $|\mathbf{H}|_{\max }$, given by (5) and (6), respectively. The magnitude of the magnetic torque that makes the MAT rotate is limited by the minimum strength of the applied magnetic field $|\mathbf{H}|_{\text {min }}$. If the MAT position lies in $\mathcal{B}$, then (5) shows that the smallest $|\mathbf{H}|_{\text {min }}$ that occurs in the ball, $\min _{\mathcal{B}}\left(|\mathbf{H}|_{\min }\right)$, happens at the position in $\mathcal{B}$ where the MAT is the farthest from the RPM and is given by

$$
\min _{\mathcal{B}}\left(|\mathbf{H}|_{\min }\right)=\frac{|\mathbf{M}|}{4 \pi(|\overline{\mathbf{p}}|+r)^{3}} .
$$

Fig. 2(b) shows four plots of $\min _{\mathcal{B}}\left(|\mathbf{H}|_{\text {min }}\right)$ with $r /|\overline{\mathbf{p}}|=$ $\{0.0,0.05,0.1,0.2\}$ normalized by $|\mathbf{M}| / 4 \pi|\overline{\mathbf{p}}|^{3}$. As $r /|\overline{\mathbf{p}}|$ increases, the worst-case field magnitude over all positions in $\mathcal{B}$ decreases, and the figure clearly shows that $\min _{\mathcal{B}}\left(|\mathbf{H}|_{\text {min }}\right)$ is invariant to changing $\bar{\theta}$. If the field magnitude is important for MAT control, then $|\overline{\mathbf{p}}|$ should be decreased to keep the worst-case magnitude acceptable. This should be done with care, however, as the attractive magnetic force between MAT and RPM increases dramatically with decreasing $|\mathbf{p}|$.

\section{Worst-case Field Rotation Speed}

The angular velocity of the applied field varies according to (7). Typically, it is necessary to ensure that the maximum applied field speed never exceeds the maximum potential rotation speed of a MAT. At any given position in the ball, the applied field rotation speed is bound from above by $|\boldsymbol{\omega}| \max$, which can be obtained by substituting $|\mathbf{H}|_{\text {min }}$ for $|\mathbf{H}|$ in (7). After substituting (5) and (6) into the resulting expression, $|\boldsymbol{\omega}|_{\max }$ becomes

$$
|\boldsymbol{\omega}|_{\max }=\sqrt{1+3|\tilde{\mathbf{p}}|^{2}}|\boldsymbol{\Omega}| .
$$

Because $\tilde{\mathbf{p}}$ is the projection of $\hat{\mathbf{p}}$ onto the plane perpendicular to $\hat{\Omega}, \max _{\mathcal{B}}\left(|\boldsymbol{\omega}|_{\max }\right)$ occurs at the position in $\mathcal{B}$ that maximizes $|\tilde{\mathbf{p}}|$, resulting in:

$$
\max _{\mathcal{B}}\left(|\boldsymbol{\omega}|_{\max }\right)=\sqrt{1+3 \sin ^{2}(\alpha)}|\boldsymbol{\Omega}|
$$

where

$$
\alpha= \begin{cases}90^{\circ} & \text { if }\left|90^{\circ}-\bar{\theta}\right| \leq \beta \\ \bar{\theta}+\beta & \text { if } \bar{\theta}<90^{\circ}-\beta \\ \bar{\theta}-\beta & \text { if } \bar{\theta}>90^{\circ}+\beta\end{cases}
$$

and $\beta$ is the angle between $\overline{\mathbf{p}}$ and every position in $\mathcal{B}$ whose vector lies tangent to the ball surface.

Fig. 2(c) shows four plots of $\max _{\mathcal{B}}\left(|\boldsymbol{\omega}|_{\max }\right)$ normalized by $|\boldsymbol{\Omega}|$, with $r /|\overline{\mathbf{p}}|=\{0.0,0.05,0.1,0.2\}$. As $r /|\overline{\mathbf{p}}|$ increases, the worst-case rotation speed tends to increase, however, $\max _{\mathcal{B}}\left(|\boldsymbol{\omega}|_{\max }\right)$ is slower near axial than radial positions. The fastest rotation speed at any position will never surpass $2|\boldsymbol{\Omega}|$ according to the point-dipole model. The worst-case speed in the radial position is already $2|\Omega|$ and cannot increase any further with increasing $r /|\overline{\mathbf{p}}|$. If maintaining field rotation speed below some threshold is critical for MAT actuation, then the RPM rotation speed should slow down as $r /|\overline{\mathbf{p}}|$ increases, and axial positions should be favored over radial. 


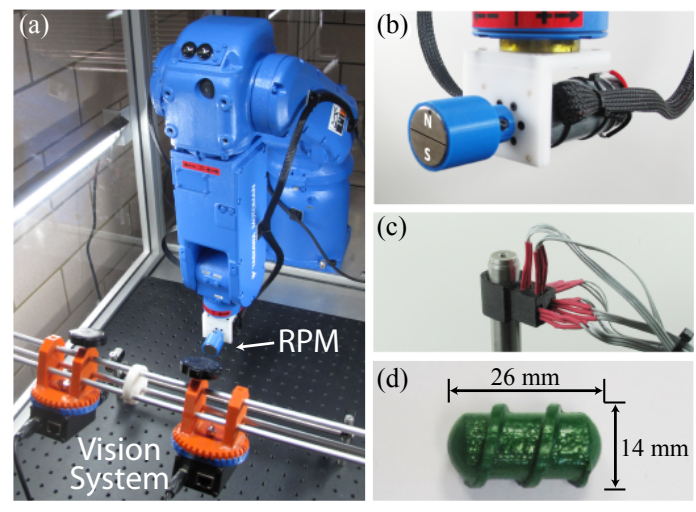

Fig. 3. The Yaskawa Motoman MH5 6-DOF robotic manipulator (a) is used to position the RPM (b). A custom, three-axis magnetic field sensor (c) and a capsule-shaped, threaded, screw MAT (d) are used to obtain experimental results. The thread of the MAT has a pitch of $9 \mathrm{~mm}$ and is $1.5 \mathrm{~mm}$ deep and the MAT contains a $6.35 \mathrm{~mm}$ cube Grade-N52 NdFeB permanent magnet positioned at the device's center-of-gravity.

\section{EXPERIMENTAl Results \& Discussion}

The RPM is positioned with a Yaskawa Motoman MH5 6-DOF robotic manipulator (Fig. 3(a)), and consists of a cylindrical $25.4 \mathrm{~mm}$ diameter, $25.4 \mathrm{~mm}$ long, Grade-N42, diametrically magnetized (i.e., along the diameter) $\mathrm{NdFeB}$ permanent magnet (Fig.3(b)) driven by a Maxon $24 \mathrm{~V}$ AMax DC motor with an Advanced Motion Controls servo control drive and amplifier. The field produced by the RPM closely follows the point-dipole model, with $|\mathbf{M}|=$ 12.7 $\mathrm{A} \cdot \mathrm{m}^{2}$, at distances where actuation typically occurs [7].

We demonstrate the theory presented in Sec. III by measuring the magnetic field generated by the RPM in the center and on the surface of two imaginary balls with $12.5 \mathrm{~mm}$ radius, centered in axial and radial positions $125 \mathrm{~mm}$ from the RPM (Fig. 4). Each ball center represents an expected position that could have been obtained from a localization system. The radius represents a potential worst-case bound for the imaginary localization system, making $r /|\overline{\mathbf{p}}|=0.1$. The magnetic field $\mathbf{H}$ is measured in terms of the magnetic flux density $\mathbf{B}$, where $\mathbf{B}=\mu_{0} \mathbf{H}$, using a three-axis sensor (Fig. 3(c)), which is discussed in [7].

For each ball, the rotating magnetic field is measured at the ball center and at six other positions that span the diameter of the ball in each coordinate direction (seven total measurements), shown at the top of Fig. 4. Figs. 4(a) and 4(c) show the magnetic field measured by the three-axis sensor at each ball center in the $\mathbf{x}-\mathbf{z}$ and $\mathbf{y}-\mathbf{z}$ planes along with the field predicted by the point-dipole model. The rotation axis (found using principal component analysis) differs from that predicted with the point-dipole model by $3.51^{\circ}$ and $2.85^{\circ}$ in the axial and radial positions, respectively. Figs. 4(b) and 4(d) show tables presenting the deviation angle $\psi$, measured from the rotation axis at each ball center, along with the minimum field magnitude $|\mathbf{B}|_{\text {min }}$ and the maximum field rotation speed $|\boldsymbol{\omega}|_{\text {max }}$, all measured at each ball center and positions $\mathbf{p}_{\mathbf{1}}$ through $\mathbf{p}_{6} \cdot|\boldsymbol{\Omega}|=2.5 \mathrm{rad} / \mathrm{s}$ in both both examples.

Sec. III-A predicts that the worst-case deviation angle of the local field rotation axis in the ball, measured from the expected local field rotation axis, is largest for axially centered balls and is smallest for radially centered balls. This is verified by Figs. 4(b) and 4(d), which show maximum deviations of $19.2^{\circ}$ for the axial ball, and $8.1^{\circ}$ for the radial ball. For a ball with $r /|\overline{\mathbf{p}}|=0.1$, Fig. 2(a) predicts that the worst case deviation in the axial and radial expected positions are $17.1^{\circ}$ and $8.6^{\circ}$, respectively. The measured deviation is greater than the predicted worst-case in the axial ball. This may be caused by error between the dipole model and the actual magnetic field, or nonidealities in the field sensor.

The worst-case (i.e., the weakest) field magnitude if the MAT is known to reside within some ball is predicted in Sec. III-B to be purely a function of the expected distance between the ball center and the RPM. Because the position where the worst-case magnitude occurs is the position in the ball farthest from the RPM, Sec. III-B can be verified by examining the minimum field magnitude at $\mathbf{p}_{\mathbf{6}}$ for the axially centered ball, and at $\mathbf{p}_{\mathbf{4}}$ for the radially centered ball. Figs. 4(b) and 4(d) show that the minimum field strength (magnetic flux density) is $0.45 \mathrm{mT}$ in both positions. The point-dipole model predicts that the minimum field strength at both positions should be $0.49 \mathrm{mT}$.

Sec. III-C predicts that the worst-case (i.e., the fastest) field rotation speed is fastest in the radially centered ball and is slowest in the axially centered ball. This is verified in Figs. 4(b) and 4(d), which show the fastest rotation speed in the axially centered ball to be $2.54 \mathrm{rad} / \mathrm{s}$ versus $5.09 \mathrm{rad} / \mathrm{s}$ in the radially centered ball. With $r /|\overline{\mathbf{p}}|=0.1$ and $|\boldsymbol{\Omega}|=$ $2.5 \mathrm{rad} / \mathrm{s}$, Fig. 2(c) predicts that the worst-case rotation speed should be $2.54 \mathrm{rad} / \mathrm{s}$ and $5.00 \mathrm{rad} / \mathrm{s}$ in the axially and radially centered balls, respectively.

With known localization uncertainty, the RPM should be operated so that the factors presented in Sec. III are within bounds tolerated by the MAT. For a constant uncertainty bound $r$, increasing $|\overline{\mathbf{p}}|$ decreases $r /|\overline{\mathbf{p}}|$, and decreases the worst-case rotation axis misalignment and rotation speed. However, increasing $|\overline{\mathbf{p}}|$ decreases the worst-case field magnitude, which is typically required to be above some threshold to maintain control authority. Additionally, the worst-case rotation axis is minimized in a radial position, and the worstcase rotation speed is minimized in an axial position. These factors must be considered during RPM motion planning.

Scaling the dimensions of the RPM can mitigate effects due to localization uncertainty. The field generated by an RPM scales homothetically, meaning that the field of an RPM whose dimensions have been scaled by a factor $s$ (the RPM volume is scaled by $s^{3}$ ), measured at the position $s \mathbf{p}$, is the same as that of an unscaled RPM measured at the position $\mathbf{p}$. If the localization system and the uncertainty bound $r$ are invariant to RPM scaling, operating the MAT at $s \mathbf{p}$ makes the expected position become $s \overline{\mathbf{p}}$, and the relative uncertainty becomes $r /(s|\overline{\mathbf{p}}|)$, which scales as $s^{-1}$. Therefore, scaling the dimensions of the RPM by $s$ and operating at equivalently scaled distances, without changing $r$, decreases $r /(s|\overline{\mathbf{p}}|)$ along with the worst-case rotation axis misalignment, field magnitude, and rotation speed. 


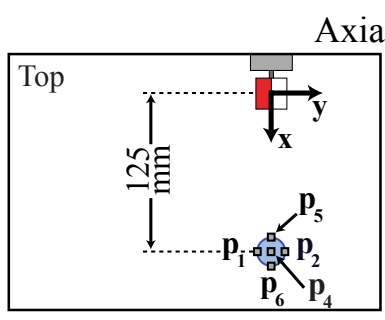

(a)

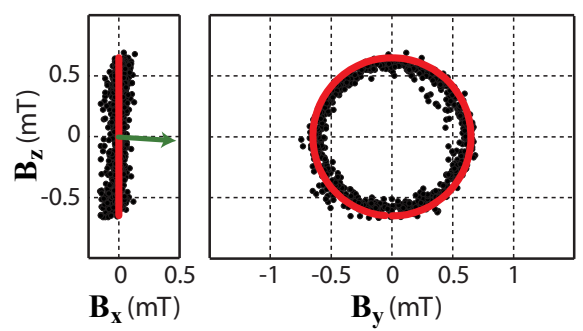

(b)

\begin{tabular}{|c|c|c|c|}
\hline & $\psi(\mathrm{deg})$ & $|\mathbf{B}| \min (\mathrm{mT})$ & $|\boldsymbol{\omega}|_{\max }(\mathrm{rad} / \mathrm{s})$ \\
\hline \hline $\mathbf{p}_{\mathbf{0}}$ & - & 0.61 & 2.51 \\
\hline \hline $\mathbf{p}_{\mathbf{1}}$ & 18.1 & 0.60 & 2.52 \\
\hline $\mathbf{p}_{\mathbf{2}}$ & 19.2 & 0.59 & 2.50 \\
\hline $\mathbf{p}_{\mathbf{3}}$ & 19.1 & 0.61 & 2.54 \\
\hline $\mathbf{p}_{\mathbf{4}}$ & 19.0 & 0.60 & 2.51 \\
\hline $\mathbf{p}_{\mathbf{5}}$ & 1.8 & 0.83 & 2.52 \\
\hline $\mathbf{p}_{\mathbf{6}}$ & 1.7 & 0.45 & 2.51 \\
\hline
\end{tabular}

Radial Ball

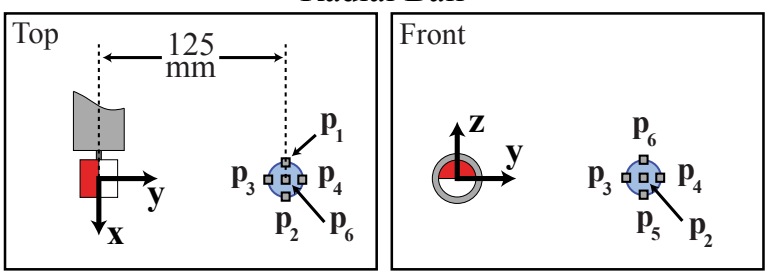

(c)
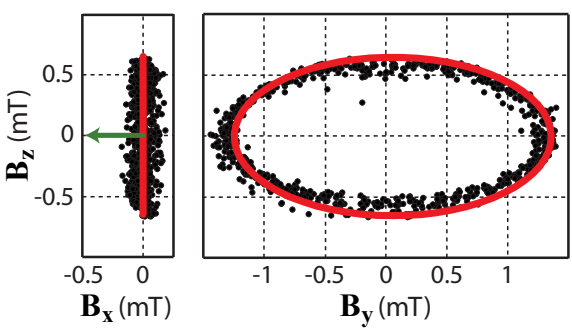

(d)

\begin{tabular}{|c|c|c|c|}
\hline & $\psi$ (deg) & $|\mathbf{B}| \min (\mathrm{mT})$ & $|\boldsymbol{\omega}|_{\max }(\mathrm{rad} / \mathrm{s})$ \\
\hline \hline $\mathbf{p}_{\mathbf{0}}$ & - & 0.59 & 4.85 \\
\hline \hline $\mathbf{p}_{\mathbf{1}}$ & 8.1 & 0.58 & 5.09 \\
\hline $\mathbf{p}_{\mathbf{2}}$ & 7.5 & 0.59 & 5.00 \\
\hline $\mathbf{p}_{\mathbf{3}}$ & 0.2 & 0.81 & 5.04 \\
\hline $\mathbf{p}_{\mathbf{4}}$ & 0.1 & 0.45 & 5.05 \\
\hline $\mathbf{p}_{\mathbf{5}}$ & 0.7 & 0.58 & 5.01 \\
\hline $\mathbf{p}_{\mathbf{6}}$ & 0.6 & 0.59 & 4.93 \\
\hline
\end{tabular}

Fig. 4. The theory presented herein is verfied by measuring the magnetic field (magnetic flux density) in the center and on the surface of two imaginary balls (shown in the scale drawings at the top of the figure). Both balls have radius $12.5 \mathrm{~mm}$ and their centers (denoted by $\mathbf{p}_{\mathbf{0}}$ ) are $125 \mathrm{~mm}$ away from the RPM. The measured and predicted rotating field at both ball centers is shown in (a) and (c) for the axial and radial examples, respectively. Tables (b) and (d) show the rotation axis deviation $\psi$ from that measured at po, the minimum field strength $|\mathbf{B}|$ min measured, and maximum rotation speed $|\boldsymbol{\omega}|$ max measured at varying positions on each ball.

Unlike uniform magnetic fields, nonuniform fields (such as those produced by the RPM) cause a magnetic force to be applied to the MAT. As demonstrated in [7], the magnetic force can assist or hinder MAT actuation. Magnetic force may substantially impact successful MAT actuation in the presence of localization uncertainty. If the magnetic force opposes the desired direction of motion, then the MAT will be less robust to localization error than expected. If the magnetic force assists motion, then the magnetic force makes MAT actuation more robust under uncertainty in some positions and produces no improvement in others.

We demonstrate the above by actuating a threaded, capsule-shaped MAT (Fig. 3(d)) within a clear PVC lumen with $22.2 \mathrm{~mm}$ inner diameter, lightly lubricated with personal lubricant jelly. While the MAT travels through the lumen, the RPM position is visually servoed to maintain a radial position $\overline{\mathbf{p}}=[0,0,-125]^{\top} \mathrm{mm}$ in Figs. 5(a) and 5(b), where magnetic force neither assists nor hinders motion, and a leading position $\overline{\mathbf{p}}=[0,72.2,-102]^{\top} \mathrm{mm}$ in Figs. 5(c) and 5(d), where magnetic force assists motion, using a stereo vision system (detailed in [7]) producing millimeter tracking accuracy at 30 frames-per-second (Fig. 3(a)). In both positions, $|\overline{\mathbf{p}}|=125 \mathrm{~mm}$ and $\hat{\mathbf{\Omega}}$ is oriented to keep the rotation axis of the field at the MAT position aligned in the negative $\mathbf{y}$ direction. Localization uncertainty is artificially induced by physically blocking the MAT from view of the vision system. When sight is lost, the vision system assumes the MAT to be in the last known position. This represents uncertainty due to localization failure, however, the effect is the same for uncertainty due to noise or slow sampling frequency (i.e., the MAT is not where expected).

Figs. 5(a) and 5(c) show the MAT being actuated with $|\boldsymbol{\Omega}|=2.5 \mathrm{rad} / \mathrm{s}$ in the radial and leading positions, respectively. In both examples, the MAT begins within sight of the vision system at $t=0 \mathrm{~s}$. The MAT passes beneath the visual obstruction at $t=48 \mathrm{~s}$ and $t=30 \mathrm{~s}$ for the radial and leading positions, respectively, where the vision system loses sight of the MAT. In the radial case, the magnetic force opposes the desired MAT motion while the MAT continues down the lumen. The $30 \mathrm{~mm}$ long obstruction is the largest the MAT is capable of tolerating without the combination of reduced field strength and change in field rotation axis causing stepout. In the leading case, the magnetic force assists the MAT through the obstruction and the MAT easily passes through.

Figs. 5(b) and 5(d) show the MAT being actuated with $|\boldsymbol{\Omega}|=0.5 \mathrm{rad} / \mathrm{s}$. At this speed, the MAT in the radial position passes through the $60 \mathrm{~mm}$ obstruction (Figs. 5(b)) despite the field magnitude decreasing and the field rotation axis pitching $39.1^{\circ}$ from expected at the last known position. In the leading position, the MAT is pulled through the obstacle until it steps-out at $t=338 \mathrm{~s}$. At this time, the field strength is larger than when the RPM is in sight, however, the field rotation axis is pitched $60.2^{\circ}$ from expected (and desired) at the last known position, likely causing the RPM to lose control authority. Clearly, applying magnetic force to assist motion 


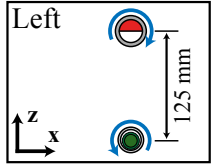

(a) $2.5 \mathrm{rad} / \mathrm{s}$
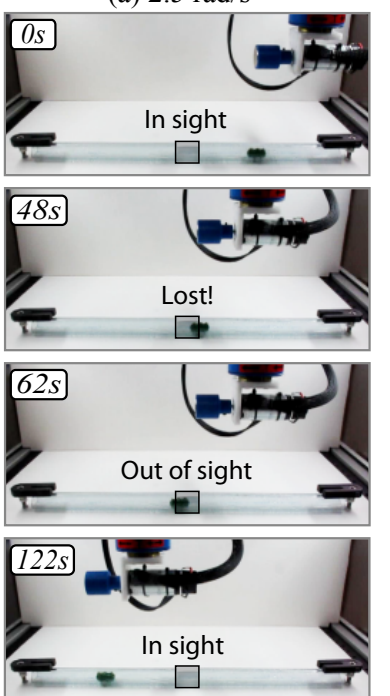
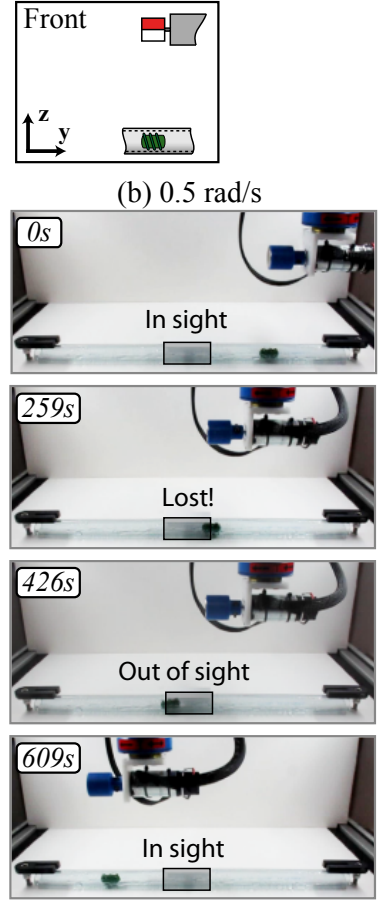

(b) $0.5 \mathrm{rad} / \mathrm{s}$
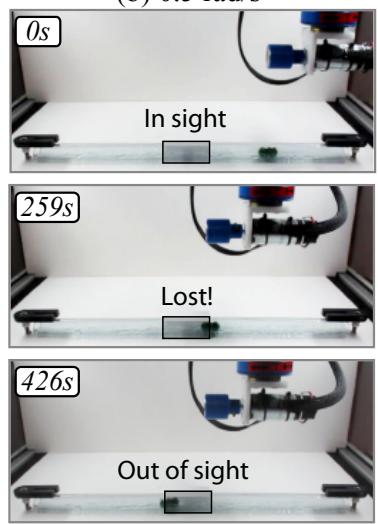

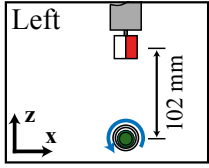

(c) $2.5 \mathrm{rad} / \mathrm{s}$
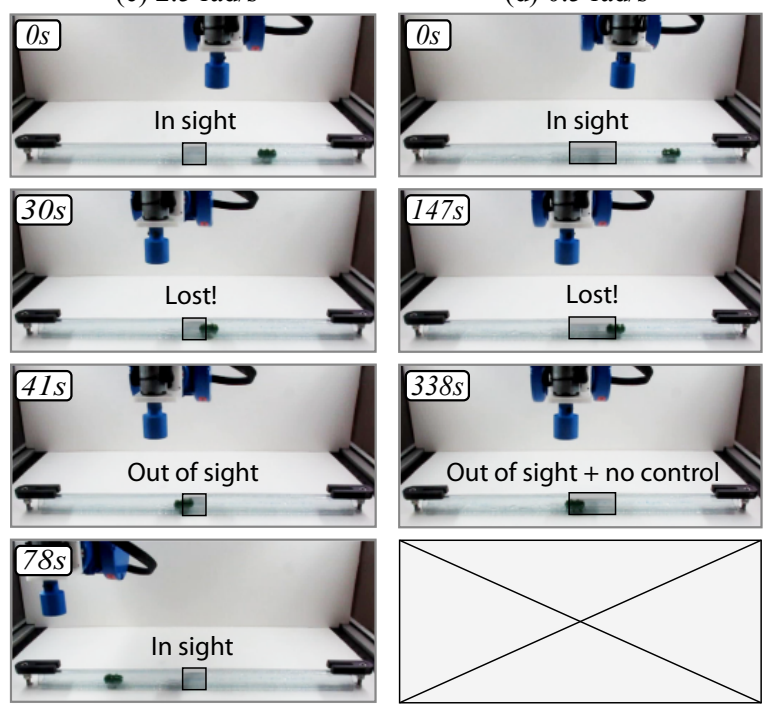

Fig. 5. The capsule MAT (Fig. 3(d)) is actuated in a radial position with $\overline{\mathbf{p}}=[0,0,-125]^{\top} \mathrm{mm}$ at $2.5 \mathrm{rad} / \mathrm{s}$ (a) and $0.5 \mathrm{rad} / \mathrm{s}$ (b), and in an unintuitive leading position with $\overline{\mathbf{p}}=[0,72.2,-102]^{\top} \mathrm{mm}$ at $2.5 \mathrm{rad} / \mathrm{s}$ (c) and $0.5 \mathrm{rad} / \mathrm{s}$ (d). In the radial position, the magnetic force neither assists or opposes the desired MAT motion. In the leading position, the magnetic force assists desired motion. The MAT is driven through a region (shaded in the images) $30 \mathrm{~mm}$ long in (a) and (c), and $60 \mathrm{~mm}$ long in (b) and (d), where the MAT is blocked from sight of the vision tracking system to induce uncertainty.

does not guarantee successful actuation, and as demonstrated by the successful actuation in the radial example, where the magnetic force opposed motion, an assistive magnetic force may not be useful in some positions. Scaling the RPM dimensions by $s$ and operating at equivalently scaled distances scales the magnetic force by $s^{-1}$ for both helping and hindering forces. Fully understanding the influence of magnetic force is extremely important for robust actuation, and is the topic of future work.

\section{CONCLUSION}

Any system for MAT localization will produce an expected MAT position with an associated uncertainty. Unlike actuation with uniform magnetic fields produced by arrangements of electromagnets, in the nonuniform field produced by a single RPM, the applied field rotation axis, field magnitude, and instantaneous rotational velocity change as the MAT position varies. If the RPM position and rotation axis are selected according to an expected MAT position, deviation of the actual MAT position from expected (caused by sensor uncertainty) may cause undesired behavior. In this paper, we have developed and experimentally verified worst-case bounds on the applied field rotation axis, field magnitude, and instantaneous rotational velocity from those expected, given a worst-case bound on localization error. These bounds can be used to design RPM operating procedures that reduce undesired MAT behavior given known localization uncertainty. The results apply to any rotating MAT, actuated by a single RPM, including active capsule endoscopes and magnetic microrobots.

\section{REFERENCES}

[1] B. J. Nelson, I. K. Kaliakatsos, and J. J. Abbott, "Microrobots for minimally invasive medicine," Аппи. Rev. Biomed. Eng., vol. 12, pp. 55-85, 2010.

[2] J. L. Toennies, G. Tortora, M. Simi, P. Valdastri, and R. J. Webster III, "Swallowable medical devices for diagnosis and surgery: the state of the art," J. Mech. Eng. Sci., vol. 224, no. 7, pp. 1397-1414, 2010.

[3] G. Ciuti, P. Valdastri, A. Menciassi, and P. Dario, "Robotic magnetic steering and locomotion of capsule endoscope for diagnostic and surgical endoluminal procedures," Robotica, vol. 28, no. 2, pp. 199207, 2010.

[4] S. Yim and M. Sitti, "Design and analysis of a magnetically actuated and compliant capsule endoscopic robot," in Proc. IEEE Int. Conf. Robotics and Automation, 2011, pp. 4810-4815.

[5] A. W. Mahoney and J. J. Abbott, "Managing magnetic force applied to a magnetic device by a rotating dipole field," Appl. Phys. Lett., vol. 99, no. 134103, pp. 1-3, 2011.

[6] T. W. R. Fountain, P. V. Kailat, and J. J. Abbott, "Wireless control of magnetic helical microrobots using a rotating-permanent-magnet manipulator," in Proc. IEEE Int. Conf. Robotics and Automation, 2010, pp. $576-581$.

[7] A. W. Mahoney, D. L. Cowan, K. M. Miller, and J. J. Abbott, "Control of untethered magnetically actuated tools using a rotating permanent magnet in any position," in Proc. IEEE Int. Conf. Robotics and Automation, 2012.

[8] D. Fischer, R. Schreiber, D. Levi, and R. Eliakim, "Capsule endoscopy: the localization system," Gastrointest. Endoscopy Clin. N. Am., vol. 14, no. 1, pp. 25-31, 2004.

[9] M. Salerno, G. Ciuti, G. Lucarini, R. Rizzo, P. Valdastri, A. Menciassi, A. Landi, and P. Dario, "A discrete-time localization method for capsule endoscopy based on on-board magnetic sensing," Meas. Sci. Technol., vol. 23, no. 01570, 2012.

[10] M.-G. Kim, Y.-S. Hong, and E.-J. Lim, "Position and orientation detection of capsule endoscopes in spiral motion," Int. J. of Prec. Eng. and Manuf., vol. 11, no. 1, pp. 31-37, 2010.

[11] F. Carpi and C. Pappone, "Magnetic maneuvering of endoscopic capsules by means of a robotic navigation system," IEEE Trans. Biomed. Eng., vol. 56, no. 5, pp. 1482-1490, may 2009. 